\title{
Geobacillus stearothermophilus LV cadA gene mediates resistance to cadmium, lead and zinc in zntA mutants of Salmonella enterica serovar Typhimurium
}

\author{
JOSÉ M PÉREZ1 ${ }^{1}$, GONZALO A PRADENAS ${ }^{1}$, CLAUDIO A NAVARRO $^{1}$, \\ DANIEL R HENRÍQUEZ ${ }^{1}$, SERGIO E PICHUANTES ${ }^{2}$ and CLAUDIO C VÁSQUEZ1,*
}

\author{
${ }^{1}$ Laboratorio de Microbiología Molecular, Departamento de Biología, Facultad de Química y Biología, \\ Universidad de Santiago de Chile, Alameda 3363, Santiago, Chile \\ ${ }^{2}$ Blood Testing Division, Chiron Corporation, Emeryville, CA, USA
}

\begin{abstract}
Salmonella enterica serovar Typhimurium cells expressing the cadA gene of Geobacillus stearothermophilus LV exhibit a hypersensitive phenotype to cadmium chloride. Deletion of the ORF STM3576 from the Salmonella genome resulted in cadmium, lead and zinc sensitivity, confirming that this ORF is a homologue of the zntA gene. The observed sensitivity was reverted upon expression of the G. stearothermophilus LV $c a d A$ gene. These results indicate that the $c a d A$ gene product is involved in $\mathrm{Cd}, \mathrm{Pb}$ and $\mathrm{Zn}$ resistance as a classical P-type ATPase and strongly suggest that the observed hypersensitive phenotype to these metals can be related to the function of the host $z n t A$ gene product.
\end{abstract}

Key terms: CadA, cadmium, lead, P-type ATPase, zinc, ZntA

\section{INTRODUCTION}

When present at appropriate amounts some heavy metals are essential elements for the cell metabolism. At higher concentrations, however, they can display severe toxic effects forcing the cell to maintain an exquisite surveillance on the intracellular levels of these ions.

A group of heavy metals with no known biological function that are considered merely toxics at any concentration has also been reported (Silver, 1998). These metals include cadmium, lead and mercury, behave as weak Lewis acids, and can react with thiolate and/or imidazol groups of cellular proteins affecting their biological activities (Lebrun et al., 1994). The widespread distribution of $\mathrm{Cd}, \mathrm{Hg}$ and $\mathrm{Pb}$ in the environment has pushed the microbial populations to develop specific resistance systems. Preventing ion accumulation by active cation efflux and sequestering by small binding proteins analogous to eukaryotic metallothioneins are some of the mechanisms that prokaryotes have developed to tolerate high concentrations of these elements (Naz et al., 2005; Rensing et al., 1998).

The main translocating proteins involved in metal homeostasis belong to the P-type ATPase family. These proteins translocate ions from the cytoplasm by active transport using ATP-driven energy. Bacterial representative members of this family include CadA, CopA, CopB and CtaA (Apell, 2004; Nucifora et al., 1989; Rosen, 2002).

The most characterized $\mathrm{Cd}$ (and $\mathrm{Pb}$ ) resistance determinants are the CadA ATPase from the Staphylococcus aureus plasmid pI258 (Nucifora et al., 1989) and the $z$ ntA gene of E. coli (Rensing et al., 1997).

We have been interested in studying bacterial heavy metal resistance in Gram-

Corresponding author: Claudio C. Vásquez. Laboratorio de Microbiología Molecular, Facultad de Química y Biología, Universidad de Santiago de Chile, Casilla 40, Correo 33, Santiago, Chile. Phone: (56) 2- 6810357. Fax: (56) 2- 681 2108. E-mail: cvasquez@usach.cl 
positive bacteria from the soil. Geobacillus stearothermophilus LV is a thermophilic, plasmidless, sporeforming microorganism capable of growing at elevated concentrations of heavy metals (Lobos and Vásquez, 1993). We have previously isolated a DNA fragment from $G$. stearothermophilus $\mathrm{LV}$ that encodes three open reading frames (ORFs), two of them exhibiting high sequence homology and gene organization features similar to the cadA and cadC genes encoded by the $S$. aureus pI258 plasmid (Nerey et al., 2002). We also communicated that overexpression of the G. stearothermophilus LV cadA gene caused a hypersensitive phenotype to $\mathrm{Cd}$ in E. coli (Nerey et al., 2002).

Here we report that the hypersensitive phenotype previously described in cells of $E$. coli overexpressing the CadA protein is also observed in Salmonella enterica serovar Typhimurium. Using genetic and in silico analysis we found that ORF STM3576 from $S$. Typhimurium 14028 s encodes a gene that is homologous to the Escherichia coli zntA gene which has been associated with $\mathrm{Cd}, \mathrm{Pb}$ and $\mathrm{Zn}$ resistance (Rensing et al., 1997). We hypothesized that the Salmonella zntA gene could be related to the hypersensitivity phenotype generated by CadA overexpression as we did not observe the classical sensitivity to $\mathrm{Cd}, \mathrm{Pb}$ or $\mathrm{Zn}$ in $S$. Typhimurium zntA mutants overexpressing the G. stearothermophilus LV cadA gene. In fact, the observed phenotype was rather an increase in the level of resistance of Salmonella to $\mathrm{Cd}, \mathrm{Pb}$ and $\mathrm{Zn}$. These results confirm that the CadA protein of $G$. stearothermophilus $\mathrm{LV}$ participates in $\mathrm{Cd}$, $\mathrm{Pb}$ and $\mathrm{Zn}$ resistance and may also suggest that the hypersensitivity phenotype to these metals observed in wild type cells could be due to an increase in the concentration of the membrane transporters CadA and/or ZntA.

\section{MATERIALS AND METHODS}

\section{Growth conditions}

Bacteria were grown in LB broth (Sambrook et al., 1989) at $37{ }^{\circ} \mathrm{C}$ with aeration and ampicillin $(50 \mu \mathrm{g} / \mathrm{ml})$ or chloramphenicol $(25 \mu \mathrm{g} / \mathrm{ml})$ was added to the medium as required.

For lead resistance assays bacteria were grown in PTY100B medium $(0.01 \%$ yeast extract, $0.01 \%$ peptone, $0.01 \%$ tryptone and $10 \mathrm{mM}$ MES, pH 6.5) amended with the corresponding antibiotics at $37{ }^{\circ} \mathrm{C}$. When cells reached the exponential phase of growth, arabinose or glucose $(0.2 \%)$ was added to the medium and cells were incubated for an additional $1 \mathrm{~h}$ before plating. Solid media contained $2 \%$ agar.

\section{Genetic manipulations}

The elimination of ORF STM3576 from the genome of $S$. Typhimurium 14028 s was carried out as described (Datsenko and Wanner, 2000). Nucleotide primers (44 mer) overlapping the start and stop codons (bold) matching the ends of the ORF STM3576 were synthesized. These primers were $z n t A 1$, GGAGGACGTTATGTCGAC CCCTGATACCGATGGCAAAAA

ATGTGTAGGCTGGAGCTGCTTCGAA and $z n t A 2$, G G G A G A A G A T T A G T T T TTACGCAGCAAACGTAGCGCGTTGCA TATGAATATCCTCCTTAGTTCCT. The 20 bases to the 3' ends of each primer (italicized) were annealed to the 5' and 3' ends of a chloramphenicol resistance cassette flanked by the FRT sites of plasmid pKD3 (Datsenko and Wanner, 2000).

$S$. Typhimurium carrying pKD46 (Datsenko and Wanner, 2000) was grown at $30{ }^{\circ} \mathrm{C}$ in LB-ampicillin medium containing $100 \mathrm{mM}$ arabinose to an $\mathrm{OD}_{600}$ of 0.4 , made electrocompetent and transformed with $400 \mathrm{ng}$ of the PCR product. Deletions of the ORF STM3576 were confirmed by PCR using primers zntAF, GCTGTTCA TCGGCAATATCGTCTGG and $z n t A R$, CACGCTATTTCTGATTAA TGGTTTG that were complementary to flanking regions of the deleted gene.

Plasmid pBAD/CadA was constructed by insertion of the PCR-amplified $\operatorname{cadA}$ gene from $G$. stearothermophilus LV into the expression vector pBAD-TOPO (Invitrogen). Briefly, the $\operatorname{cadA}$ gene was amplified using the vector $\mathrm{pET} / \mathrm{CadA}$ as template and the primers previously described (Nerey et al., 2002). The PCR 
product was ligated to $\mathrm{pBAD}-\mathrm{TOPO}$ and cloned into E. coli Top10 as recommended by the vendor. The identity and orientation of the $\operatorname{cadA}$ gene inserted into the recombinant plasmid was confirmed by DNA sequencing and also by NcoI restriction endonuclease analysis (not shown). The purified plasmid was introduced separately into electrocompetent $S$. Typhimurium 14028 s and $S$. Typhimurium 14028s DzntA.

\section{Assay for metal sensitivity}

Cells to be tested for cadmium, zinc, lead or mercury sensitivity were grown overnight and then diluted 100-fold with fresh LB medium. $100 \mathrm{ml}$ aliquots of these dilutions were spread over $\mathrm{LB}(\mathrm{Cd}, \mathrm{Zn}$ and $\mathrm{Hg}$ ) or PYT100B agar plates $(\mathrm{Pb})$. Plates were briefly air-dried and then $15 \mu \mathrm{l}$ aliquots of 1 $\mathrm{M} \mathrm{CdCl} 2, \mathrm{ZnSO}_{4}, \mathrm{HgCl}_{2}$ or $\mathrm{PbCl}_{2}$ were spotted onto sterile disks of filter paper that had been previously placed in the center of the plates. Plates were incubated for $18 \mathrm{~h}$ at $37{ }^{\circ} \mathrm{C}$ and the area of growth inhibition zones $\left(\mathrm{cm}^{2}\right)$ was determined as described (Bauer et al., 1966).

\section{RESULTS AND DISCUSSION}

Expression of G. stearothermophilus $L V$ cadA gene results in hypersensitivity to $C d$, $\mathrm{Pb}$ and $\mathrm{Zn}$ in wild type $S$. Typhimurium

To determine whether the $G$. stearothermophilus LV cadA gene is involved in cadmium resistance in $S$. Typhimurium we cloned this gene into the arabinose-inducible $\mathrm{pBAD}$ expression vector. The recombinant plasmid was introduced by electroporation into $S$. Typhimurium. $\mathrm{CdCl}_{2}$ resistance assays were carried out in the presence of arabinose or glucose (repressor).

Figure 1 shows that expression of the $G$. stearothermophilus LV cadA gene generates a hypersensitive phenotype to $\mathrm{Cd}$ in Salmonella. This phenotype is similar to that previously observed in $E$. coli expressing the plasmid $\mathrm{pET} / \mathrm{CadA}$ (Nerey et al., 2002) and in Saccharomyces cerevisiae expressing the Listeria monocytogenes cadA gene (Wu et al., 2004).

In the presence of glucose cells carrying pBAD or $\mathrm{pBAD} / \mathrm{CadA}$ showed about the same resistance to $\mathrm{CdCl}_{2}$. These results confirm the direct relationship that exists between cadA overexpression and the cadmium hypersensitive phenotype (Nerey et al., 2002).

Because the G. stearothermophilus LV CadA protein shares a high sequence identity with the CadA protein from $S$. aureus (Nucifora et al., 1989; Rensing et al., 1998) we investigated whether the $G$. stearothermophilus $\mathrm{LV}$ CadA was also responsible for lead, zinc or mercury hypersensitivity in $S$. Typhimurium.

Upon induction, $S$. Typhimurium 14028 s carrying pBAD/CadA display an important increase in sensitivity to $\mathrm{PbCl}_{2}$ and $\mathrm{ZnSO}_{4}$ while no effect is observed with $\mathrm{HgCl}_{2}$ (Fig. 1). Conversely, when glucose was present, no sensitivity for any of these metals was observed in cells carrying $\mathrm{pBAD} / \mathrm{CadA}$. These results indicate that the observed hypersensitive phenotypes in Salmonella are a consequence of the expression of the G. stearothermophilus LV cadA gene supporting the idea that apart from $\mathrm{Cd}, \mathrm{CadA}$ is also involved in $\mathrm{Pb}$ and $\mathrm{Zn}$ transport as it has been shown for other CadA translocases (Lebrun et al., 1994; Nucifora et al., 1989).

To our knowledge, mercury ions are not transported by CadA. The fact that $G$. stearothermophilus LV cadA expression had no effect on $\mathrm{Hg}$ resistance in Salmonella favors the idea that the toxic effect produced by the overexpression of cadA in Salmonella is due mostly to an increase of metal transport and is not a consequence of a membrane instability effect. This last possibility would have probably involved a generalized hypersensitivity effect to several metals including mercury.

\section{The S. Typhimurium genome encodes a putative zntA gene}

To evaluate if the hypersensitive phenotype generated by cadA expression is related to an increase in the concentration of general 


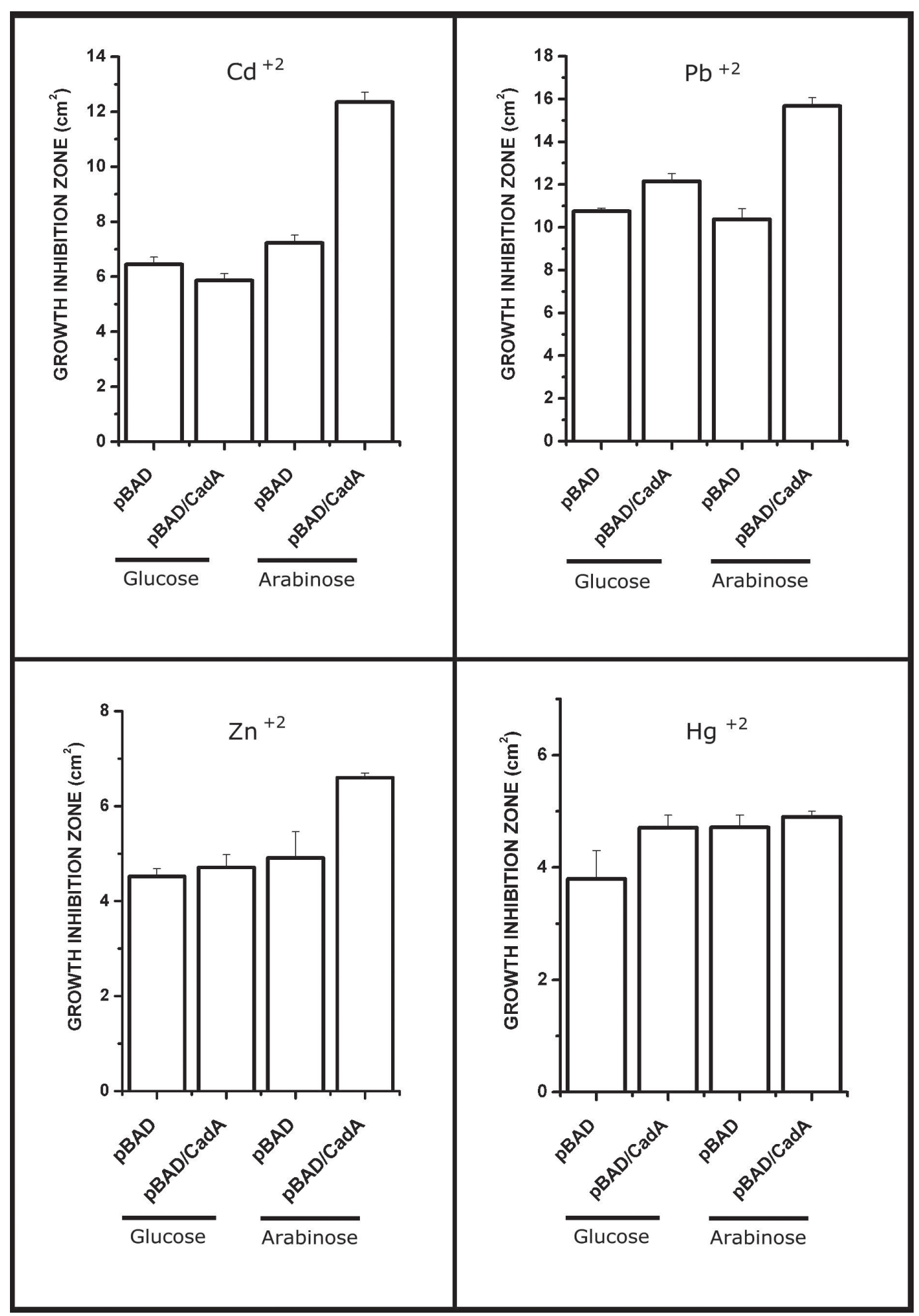

Figure 1: Expression of cadA gene from G. stearothermophilus LV generates sensitivity to $\mathbf{C d}^{+2}, \mathbf{P b}^{+2}$ and to $\mathbf{Z n}^{+2}$ in Salmonella. The growth inhibition zones $\left(\right.$ area, $\mathrm{cm}^{2}$ ) generated by $\mathrm{Cd}^{+2}$, $\mathrm{Pb}^{2+}, \mathrm{Zn}^{2+}$ and $\mathrm{Hg}^{2+}$ to $S$. Typhimurium 14028s harboring pBAD and pBAD/CadA are shown. Cells were grown in the corresponding medium plus $0.2 \%$ glucose or arabinose as required. Bars are the standard deviation $(n=4)$. 
Cd transporters we looked for $G$. stearothermophilus LV cadA homolog genes in the $S$. Typhimurium genome. Using BlastN and BlastP softwares (http:// www.ncbi.nih.gov/BLAST/) we found that $S$. Typhimurium ORF STM3576 shares 40 $\%$ identity (58\% similitude) with the $\operatorname{cadA}$ gene of $G$. stearothermophilus LV. ORF STM3576 also exhibits $76 \%$ identity with the $E$. coli zntA gene, a well-characterized P-type ATPase involved in $\mathrm{Cd}, \mathrm{Pb}$ and $\mathrm{Zn}$ transport (Hou and Mitra, 2003).

$S$. aureus CadA and E. coli ZntA proteins exhibit three highly conserved functional domains consistently present in metal-translocating P-type ATPases. A heavy metal-associated domain (HMA) is found at the amino terminal end while an E1-E2 ATPase motif is generally found between amino acids 199 and 400. The carboxy terminal end contains the hydrolase motif (Fig. 2).

A more in depth analysis of the deduced amino acid sequences revealed that all of these motifs are highly conserved in $G$. stearothermophilus LV CadA and in $S$. Typhimurium ORF STM3576 (Fig. 2).

A more detailed comparison of the three referred domains present in E. coli ZntA and in $S$. Typhimurium ORF STM3576 showed that they are about $90 \%$ identical. This high motif conservation is noteworthy since the overall identity of both proteins is only about $75 \%$.
ORF STM3576 from S. Typhimurium mediates $C d, P b$ and $\mathrm{Zn}$ resistance

To assess the effect of P-type ATPase concentration in CadA-dependent cadmium hypersensitivity, ORF STM3576 was deleted from the $S$. Typhimurium genome as described in Methods. The mutant strain was analyzed for $\mathrm{Cd}, \mathrm{Pb}$ and $\mathrm{Zn}$ resistance, metals that are known to be transported by ZntA of E. coli (Binet and Poole, 2000; Hou and Mitra, 2003) and CadA of $S$. aureus (Nucifora et al., 1989). Because $E$. coli ZntA exhibits ATPase activity in the presence of mercury (Sharma and Mitra, 2000; Mitra and Sharma, 2001) we also investigated the participation of ORF STM3576 in mercury resistance.

As shown in Figure 3, deletion of ORF STM3576 from the Salmonella genome generates a hypersensitive phenotype to $\mathrm{Cd}, \mathrm{Pb}$ and $\mathrm{Zn}$. These results coincide with those found during the analysis of sequence of the conserved domains (Fig. 2) and support the idea that the ORF STM3576 encodes a true P-type ATPase that can be associated to the transport of these metals in Salmonella. No effect was observed with mercury chloride (Fig. 3D). These findings are in apparent contradiction with the putative role assigned to the Salmonella ZntA concerning the transport of mercury ions (NCBI accession number NP_462477).

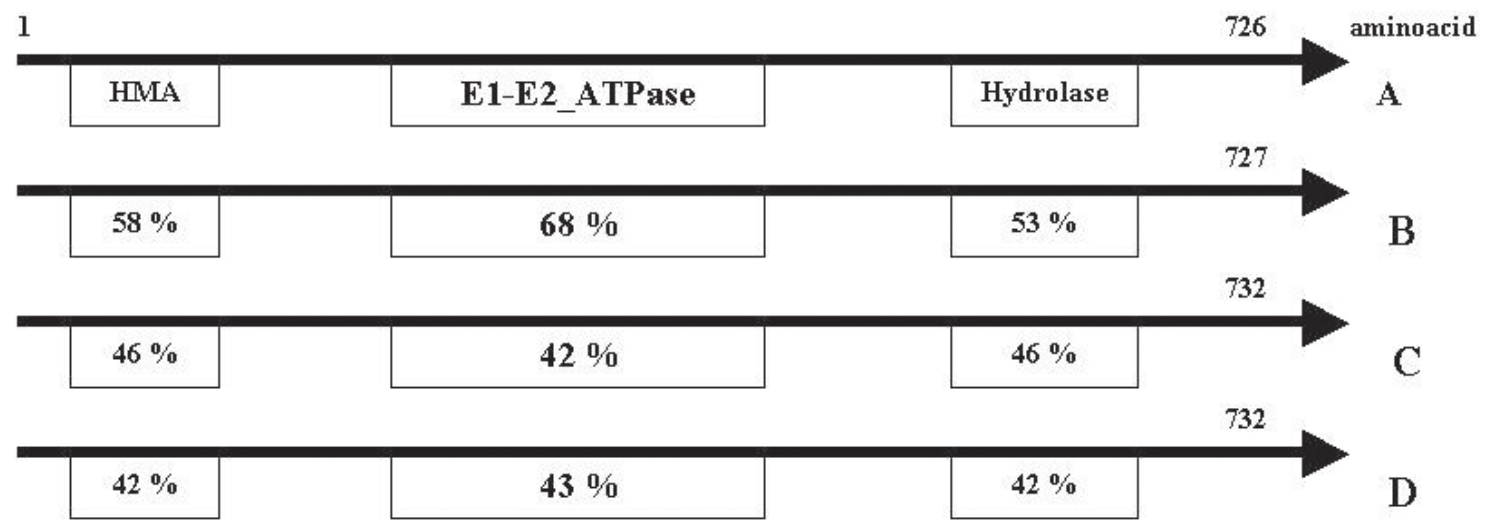

Figure 2: Comparison of HMA, ATPase and hydrolase motifs in CadA and ZntA proteins. Solid bars represent CadA from S. aureus (A) and G. stearothermophilus LV (B), ZntA from E. coli (C) and ORF STM3576 from S. Typhimurium (D). Amino acid numbers are indicated. Boxes indicate the \% of identity of each protein's domain compared to those of $S$. aureus CadA. 


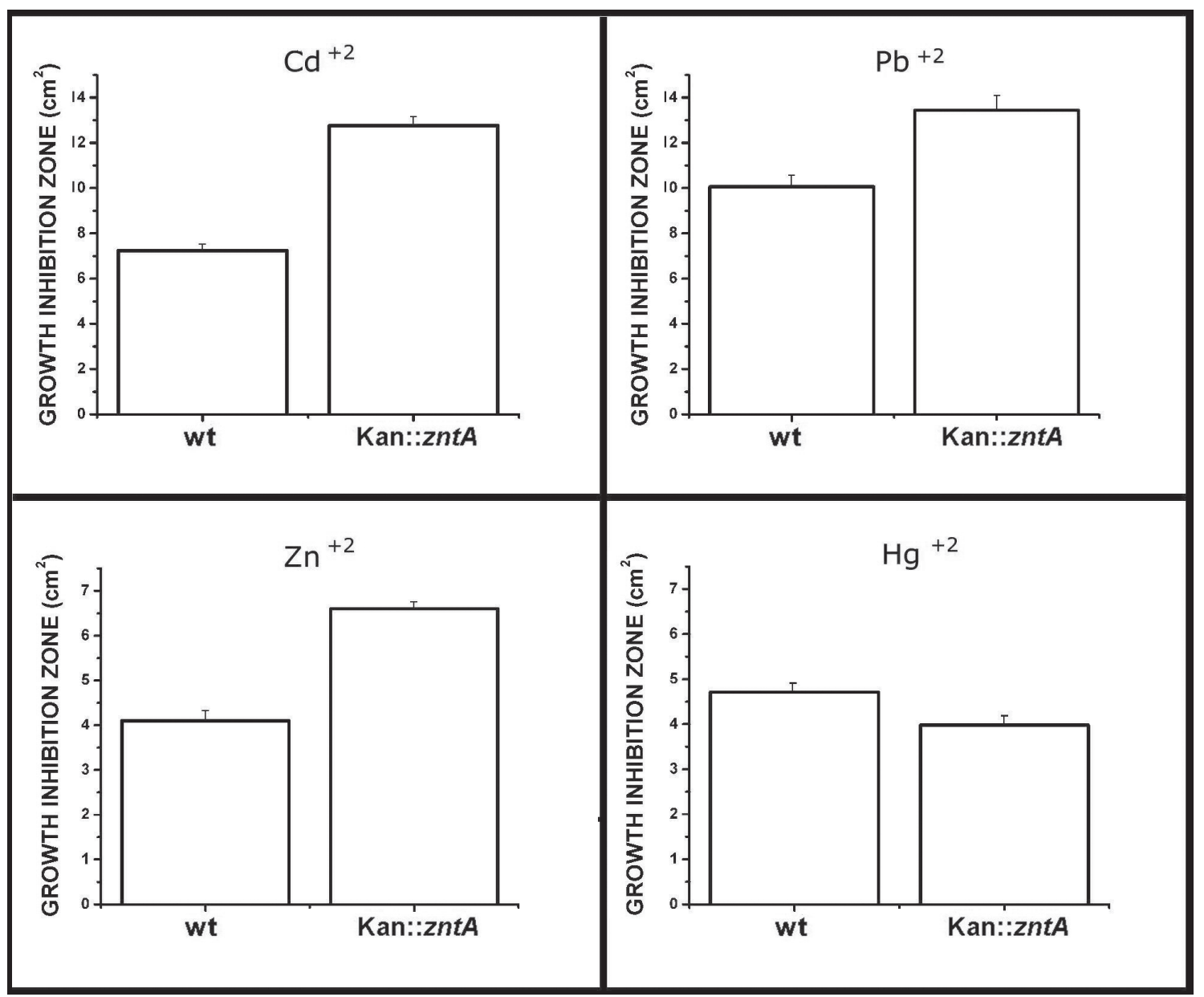

Figure 3: Deletion of ORF STM3576 generates sensitivity to $\mathrm{Cd}^{2+}, \mathrm{Pb}^{2+}$ and $\mathrm{Zn}^{2+}$ in $S$. Typhimurium. Growth inhibition zones $\left(\right.$ area, $\mathrm{cm}^{2}$ ) generated by $\mathrm{Cd}^{2+}, \mathrm{Pb}^{2+}, \mathrm{Zn}^{2+}$ and $\mathrm{Hg}^{2+}$ to $S$. Typhimurium 14028s (wt) and $S$. Typhimurium 14028s $\Delta z n t A$ (Kan::zntA). Bars represent standard deviation $(n=4)$.

Expression of $G$. stearothermophilus $L V$ cadA gene restores $\mathrm{Cd}, \mathrm{Pb}$ and $\mathrm{Zn}$ resistance in S. Typhimurium 14028s $\Delta z$ ntA

To analyze the effect of $G$. stearothermophilus LV cadA expression in resistance to $\mathrm{Cd}, \mathrm{Pb}, \mathrm{Zn}$ and $\mathrm{Hg}, S$. Typhimurium $\Delta z n t A$ cells were transformed with plasmid pBAD/CadA. As seen in Figure 4 upon CadA induction there is a complementation of the hypersensitive phenotype observed for the Kan::zntA mutant. The most obvious effect in resistance recovery was seen in Cd-treated cells. The effect on $\mathrm{Pb}$ or $\mathrm{Zn}$ resistance, although significant, was lower than that observed for $\mathrm{Cd}$. These results confirm that the cadA gene from G. stearothermophilus $\mathrm{LV}$ codes for a protein that is involved in transport of cadmium, zinc and lead as suggested from sequence comparison studies.

Several P-type ATPases have been described as result of genome sequencing. However, very little limited information is currently available about the characterization of these proteins and their participation in metal resistance. To our knowledge, there is only one report of a thermophilic microorganism P-type ATPase involved in $\mathrm{Cd}$ and $\mathrm{Zn}$ resistance (Schirawski et al., 2002). Our results also 


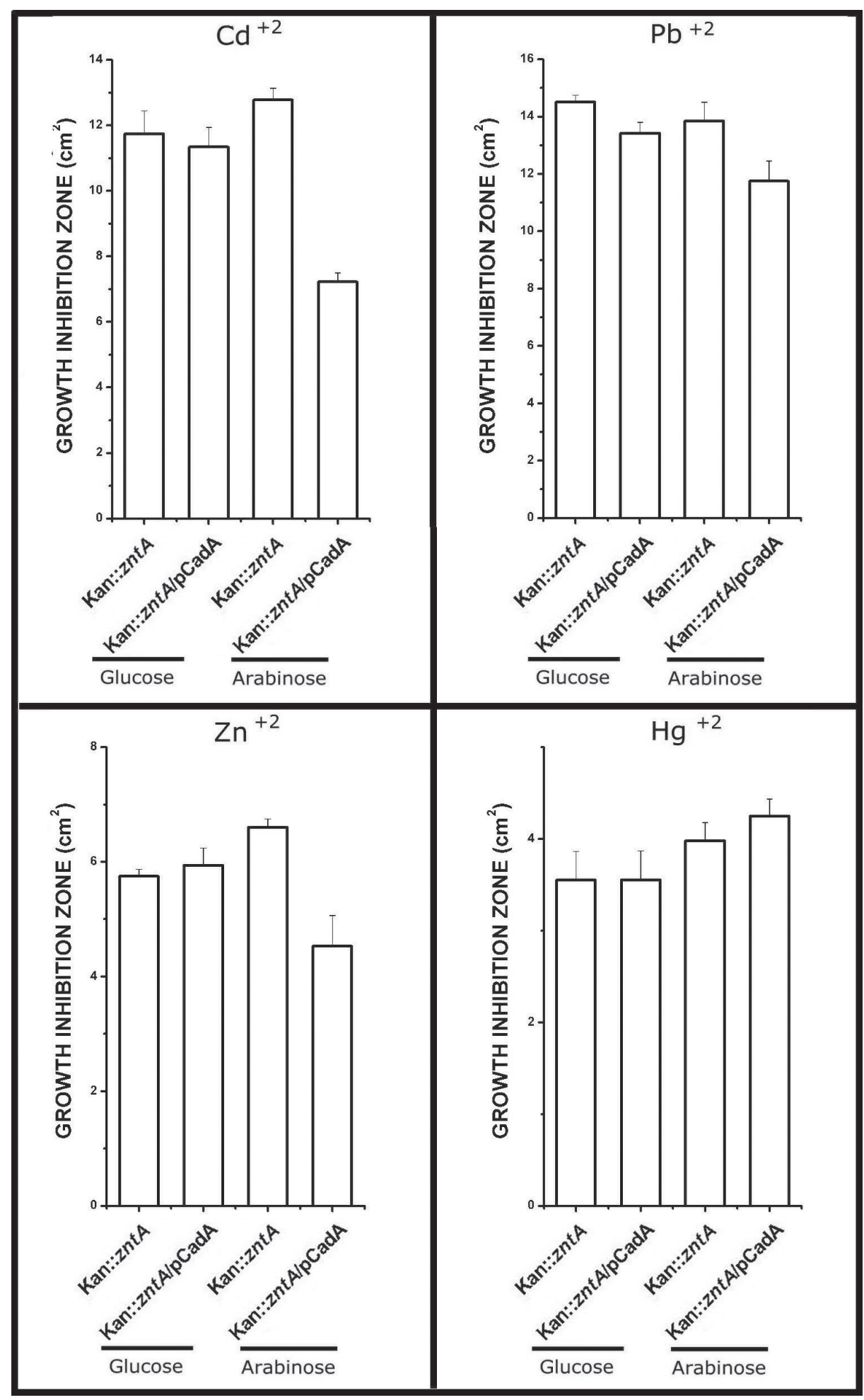

Figure 4: Expression of cadA gene from $G$. stearothermophilus $\mathrm{LV}$ mediates resistance to $\mathbf{P b}^{2+}$

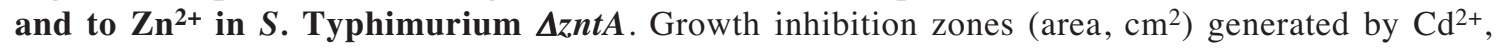
$\mathrm{Pb}^{2+}, \mathrm{Zn}^{2+}$ and $\mathrm{Hg}^{2+}$ to Salmonella Typhimurium 14028s $\Delta z n t A$ (Kan::zntA) and Salmonella Typhimurium $14028 \mathrm{~s} \Delta z n t A \mathrm{pBAD} / \mathrm{CadA}$. Cells were grown in the corresponding medium plus 0.2 $\%$ glucose or arabinose. Bars represent standard deviation $(n=4)$. 
show that the translocase from $G$. stearothermophilus LV can work with $\mathrm{PbCl}_{2}$ as well.

These findings suggest that the CadAmediated Cd sensitivity observed in Salmonella is most probably related to the expression of the heavy metal translocase encoded by ORF STM3576. Since the availability of ATP is essential for heavy metal resistance our speculation is that the observed toxicity is likely a consequence of a high local concentration of heavy metal ATP-dependent translocases generated in response to either toxic ions ( $\mathrm{ZntA})$ or by CadA overexpression in the Salmonella cell membrane.

As occurs in E. coli, probably also in Salmonella a cadmium-mediated stress would trigger a switch of aerobic to anaerobic metabolism, forcing the cell to exert a fine control on the level of ATP (Wang and Crowley, 2005). In this context, an elevated expression and/or an increased activity of ATPases, like CadA, would explain the appearance of metal hypersensitive phenotypes because of the increase in ATP consumption.

\section{ACKNOWLEDGEMENTS}

This work was supported by grants from Fondecyt (\# 1060022) and from the Dirección de Investigación, Universidad de Santiago de Chile to C. C. V. Doctoral fellowships have been awarded to C.A.N. and to D.R.H. from Conicyt and to J.M.P. from Mecesup.

\section{REFERENCES}

APELL HJ (2004) How do P-type ATPases transport ions?. Bioelectrochemistry 63: 149-156

BAUER AW, KIRBY WMM, SHERRIS JC, TURCK M (1966) Antibiotic susceptibility testing by a standardized single disk method. Am J Clin Pathol 45: 493-496

BINET M, POOLE R (2000) Cd(II), Pb(II) and Zn(II) ions regulate expression of the metal-transporting P-type ATPase ZntA in Escherichia coli. FEBS Lett 473: 6770

DATSENKO K, WANNER BL (2000) One-step inactivation of chromosomal genes in Escherichia coli K-12 using PCR products. Proc Natl Acad Sci USA 97: 6640-6645

HOU Z, MITRA B (2003) The metal specificity and selectivity of ZntA from Escherichia coli using the acylphosphate intermediate. J Biol Chem 278: 2845528461

LEBRUN M, AUDURIER A, COSSART P (1994) Plasmidborne cadmium resistance genes in Listeria monocytogenes are similar to cadA and cadC of Staphylococcus aureus and are induced by cadmium. J Bacteriol 176: 3040-3048

LOBOS C, VÁSQUEZ C (1993) Purification and characterization of $B s t \mathrm{LVI}$ restriction endonuclease, a thermostable isoschizomer of ClaI from Bacillus stearothermophilus LV. Biochim Biophys Acta 1171: 295-298

MITRA B, SHARMA R (2001) The cysteine rich aminoterminal domain of $\mathrm{ZntA}$, a $\mathrm{Pb}(\mathrm{II}) / \mathrm{Zn}(\mathrm{II}) / \mathrm{Cd}(\mathrm{II})$ translocating ATPase from Escherichia coli, is not essencial for its function. Biochemistry 40: 7694-7699

NAZ N, YOUNG H, AHMED N, GADD J (2005) Cadmium accumulation and DNA homology with metal resistance genes in sulfate-reducing bacteria. Appl Environ Microbiol 71: 4610-4618

NEREY M, PICHUANTES S, SAAVEDRA C, ARAYA M, TANTALEÁN J, VÁSQUEZ C (2002) Expression of Bacillus stearothermophilus LV cadmium resistance genes in Escherichia coli causes hypersensitivity to cadmium chloride. Curr Microbiol 45: 187-190

NUCIFORA G, CHU L, MISRA T, SILVER S (1989) Cadmium resistance from Staphylococcus aureus plasmid pI258 cadA gene results from a cadmiumefflux ATPase. Proc Natl Acad Sci USA 86: 3544-3548

RENSING C, SUN Y, MITRA B, ROSEN B (1998) Pb (II)translocating P-type ATPases. J Biol Chem 273: 32614- 32617

RENSING C, MITRA B, ROSEN B (1997) The zntA gene of Escherichia coli encodes a Zn (II)- translocating Ptype ATPase. Proc Natl Acad Sci USA 94: 1432614331

ROSEN B (2002) Transport and detoxification systems for transition metals, heavy metals and metalloids in eukaryotic and prokaryotic microbes. Comp Biochem Physiol Part A 133: 689-693

SAMBROOK J, FRITSCH EF, MANIATIS T (1989) Molecular Cloning: a laboratory manual, $2^{\text {nd }}$ Ed. Cold Spring Harbor Laboratory Press, Cold Spring Harbor, N.Y.

SCHIRAWSKI J, HAGENS W, FITZGERALD G, SINDEREN D (2002) Molecular characterization of cadmium resistance in Streptococcus thermophilus strain 4134: an example of lateral gene transfer. Appl Environ Microbiol 68: 5508-5516

SHARMA R, RENSING C, ROSEN B, MITRA B (2000) The ATP hydrolytic activity of purified $\mathrm{ZntA}$, a $\mathrm{Pb}(\mathrm{II}) /$ $\mathrm{Cd}(\mathrm{II}) / \mathrm{Zn}(\mathrm{II})$ - translocating ATPase from Escherichia coli. J Biol Chem 275: 3873-3878

SILVER S (1998) Genes for all metals- a bacterial view of the periodic table. J Ind Microbiol Biotechnol 1: 1-12

WANG A, CROWLEY D (2005) Global gene expression responses to cadmium toxicity in Escherichia coli. J Bacteriol 187: 3259-3266

WU C, BAL N, PERARD J, BOSCHERON C, MINTZ E, CATTY P (2004) A cloned prokaryotic $\mathrm{Cd}^{2+}$ P-type ATPase increases yeast sensitivity to $\mathrm{Cd}^{2+}$. Biochem Biophys Res Commun 324:1034-1040 